\section{Molecular Syndromology}

Mol Syndromol 2020;11:1-3

DOI: $10.1159 / 000506410$
Accepted: February 11, 2020

Published online: February 15, 2020

\title{
Mutated NUP188 and Other Nucleoporins as Gateways to Developmental Syndromes
}

In each eukaryotic cell, the nucleus is surrounded by the nuclear envelope (NE) in which the nuclear pore complex (NPC) is embedded. In vertebrates, the NPC is roughly $60 \mathrm{Mda}$ in size and comprises approximately 30 distinct proteins. The NPC forms an octagonal channel across the NE and functions in nucleocytoplasmic transport, maintaining the nuclear framework, and regulation of gene expression [Nofrini et al., 2016]. The number of NPCs per nucleus varies with environmental conditions and cellular activity, such that it increases during interphase and reaches a peak during the S-phase of the cell cycle. The NPC is formed by the nucleoporins (NUPs) and consists of 4 concentric cylinders, which surround an inner channel of 40-50 $\mathrm{nm}$ in diameter. NUPs are named after their molecular weight and fall into 6 main categories [Hoelz et al., 2011]. First, the transmembrane NUPs, NUP210, NDC1, and POM121, which are integral membrane proteins. They are thought to participate in NPC assembly and anchoring to the NE. Second, the membrane-apposed coat NUPs, which include NUP160, NUP133, NUP107, NUP96, NUP85, SEH1L, NUP43, NUP37, and SEC13. They form the nonameric Nup107160 complex that is required for early steps in NPC assembly. The ELYS protein is thought to be the tenth member of this Nup107-160 complex. Third, the adapter NUPs, NUP205, NUP188, NUP155, NUP93, and NUP35, which constitute the NUP93 complex. NUP188 and NUP93 control the organization of the subcompartments of the NE, such as the outer nuclear membrane, which is contiguous with the endoplasmic reticulum, and the inner nuclear membrane. NUP188 and NUP93 form a bar-
๑) 2020 S. Karger AG, Basel rier that prevents membrane proteins from passing from the outer nuclear membrane to the inner nuclear membrane [Busayavalasa et al., 2012]. Fourth, the channel NUPs, NUP62, NUP58 (alias NUPL1), and its splice variants NUP45 and NUP54, constitute the innermost cylinder of the NPC. NUP54/NUP58 are thought to form a midplane ring that undergoes reversible expansion and contraction to regulate nucleocytoplasmic traffic [Solmaz et al., 2011]. Fifth, the nuclear basket NUPs, NUP153, TPR, and NUP50, which are involved in nuclear import/ export processes [Wälde and Kehlenbach, 2010]. Specifically, NUP153 and NUP50 form a ring, which facilitates nucleocytoplasmic transport [Hoelz et al., 2011]. The NUP50 protein is highly mobile and its dynamics depends upon active RNA polymerase II-mediated transcription. In addition, depletion of Nup50 inhibits differentiation of myoblasts into myotubes [Buchwalter et al., 2014]. Sixth, the cytoplasmic filaments emerging from the NPC are composed of 7 proteins, RANBP2 (alias NUP358), NUP214, NUP98, NUP88, ALADIN, NUPL2 (alias hCG1), and RAE1, and provide interaction sites for the nucleocytoplasmic transport [Hoelz et al., 2011]. Two other members of the NPC, the mRNA export factor GLE1 and the DEAD-box-containing RNA helicase DDX19, are involved in mRNA trafficking.

Some NUPs have been related to immune disease, viral infections, and malignant disease [Nofrini et al., 2016]. Since the NUPs perform in concert essential functions, which are pivotal to the functioning of each body cell, it is difficult to conceive that germline mutations in the genes encoding NUPs may be compatible with life. Nev-
Martin Poot
Department of Human Genetics, University of Würzburg
Biozentrum, Am Hubland
DE-97074 Würzburg (Germany)
Martin_Poot@ hotmail.com 
ertheless, by analysis of multiplex consanguineous families via whole-exome sequencing, patients with mutations in the ubiquitously expressed NUP107 gene have been found [Alazami et al., 2015]. This gene also turned out to be mutated in patients with childhood-onset steroid-resistant focal segmental glomerulosclerosis [Miyake et al., 2015; Rosti et al., 2017]. In 3 out of 4 affected individuals, the mutations hampered NUP107 binding to NUP133 and NUP107 incorporation into NPCs in vitro [Miyake et al., 2015]. In addition, NUP155 insufficiency has been related to atrial fibrillation, and bialllelic NUP88 mutations have been found in patients with fetal akinesia [Bonnin et al., 2018; Preston et al., 2018]. In a 9-year-old boy with focal segmental glomerular sclerosis, a biallelic intronic variant in NUP93 was detected [Rossanti et al., 2019]. RNA sequencing and in vitro splicing assays showed that this variant caused skipping of exon 20 . The resulting protein localized in small punctate vesicles in the cytoplasm. This finding underscores that this variant is pathogenic [Rossanti et al., 2019].

In patients with Galloway-Mowat syndrome-8, an autosomal recessive disorder characterized by impaired psychomotor development, poor overall growth with microcephaly, and early-onset progressive nephrotic syndrome associated with focal segmental glomerulosclerosis, homozygous splicing mutations in NUP133 have been found [Fujita et al., 2018]. A biallelic mutation in NUP133 can also cause nephrotic syndrome type 18 (NPHS18; 618177) [Fujita et al., 2018]. Some patients may experience seizures, and some may die during childhood. In a patient with microcephaly and steroid-resistant nephrotic disorder akin to Galloway-Mowat syndrome, a homozygous mutation in NUP107 was detected [Rosti et al., 2017]. In a consanguineous family, the 4 members presenting with severe neonatal hypotonia, profound global developmental delay, progressive microcephaly, and early death carried a novel homozygous missense variant in the NUP214 gene [Shamseldin et al., 2019]. In patientderived fibroblasts, nuclei showed a dysmorphic appearance, which was previously described in NUP214 knockdown cells. In addition, the typical rim staining of NUP214 is largely displaced, which further corroborates the deleterious effect of this variant. These studies indicated that NUPs are crucial for proper development of the brain, heart, and kidneys. Yet, no clear-cut pathogenic pattern, letting alone a syndrome, seems to emerge.

The NUP188 protein, a component of the NUP93 adapter complex, received particular interest although, with NUP205, it is dispensable for NPC formation [Theerthagiri et al., 2010]. In the absence of NUP188, nuclei in- crease in size, which is due to an accelerated translocation of integral membrane proteins through NPCs. This suggests that NUP188 controls the passage of membrane proteins through NPC [Theerthagiri et al., 2010]. NUP188 also regulates chromosome segregation by localizing with its C-terminal region to the spindle poles during mitosis [Itoh et al., 2013]. Thus, in NUP188-depleted mitotic cells, chromosomes fail to align to the metaphase plate, which activates the spindle assembly checkpoint and causes subsequent mitotic arrest [Itoh et al., 2013]. The finding of a duplication affecting NUP188 and 5 neighboring genes in a cohort of 262 individuals with heterotaxy, a congenital heart disease resulting from abnormalities in left-right (LR) body patterning, prompted functional studies [Fakhro et al., 2011]. Morpholino knockdown of Nup188 in Xenopus tropicalis disrupted both morphological LR development and expression of pitx2, a molecular marker of LR patterning. Searching for a molecular mechanism for the gene dosage effect of NUP188, the authors performed knockdown of Nup188 and its binding partner Nup93 in Xenopus tropicalis [Del Viso et al., 2016]. This treatment lead to a loss of cilia during embryonic development, while leaving the NPC function largely intact [Del Viso et al. 2016]. In the Xenopus epidermis, endogenous NUP188 and NUP93 localized to the base of cilia and at centrioles. Based on these findings, the authors suggest that the organization and function of NUPs are context dependent and that they are required for the development of the structure of the heart. In a patient and his affected mother with mitral valve prolapse from an independent cohort of 55 cases, a heterozygous consensus splice donor variant was identified in NUP188 [Haskell et al., 2017]. This mutation results in a truncated protein, which lacks its C-terminal phosphorylation and ubiquitination sites. In both cases, an autosomal dominant mechanism is apparent [Fakhro et al., 2011; Haskell et al., 2017]. Dosage alterations of genes encoding proteins that bind to other proteins provoke a dominant effect [Birchler and Veitia, 2012; Poot and Haaf, 2015]. This is in agreement with the finding that NUP188 is part of the NUP93 complex [Hoelz et al., 2011].

Recently 2 patients from 2 ethnically distinct populations with 2 different homozygous nonsense gene variants of NUP188, p.Tyr96* and p.Gln113*, have been described [Sandestig et al., 2020]. These truncating mutations most likely provoke nonsense-mediated decay of the mRNA resulting in insufficiency of the NUP188 protein, thus giving rise to a circumscribed syndrome [Sandestig et al., 2020]. The 2 patients shared pre- and postnatal microcephaly, trigonocephaly, congenital bilateral 
cataracts, microphthalmia, palatal anomalies (i.e., cleft lip and palate or high-arched palate), camptodactyly, rockerbottom feet, heart anomalies, specific brain changes (such as loss of periventricular white matter or delayed myelinization), and a thin corpus callosum. Even their facial features, such as laterally extended arched eyebrows, a wide convex nose with a wide prominent nasal bridge, and prominent angulated antihelix were strikingly similar. Their lifespan (67 and 140 days) and their cause of death, central respiratory failure, were akin.

Although the number of reports on patients with mutated NUP188 is still limited, a few ramifications emerge. First, the mutations have been found by a "reverse genetics" approach. Rather than working from a presumed syndrome, clinical geneticists first searched for an altered genotype and then interpreted the phenotypes of the patient. The consensus procedure for "reverse genetics" is to perform array-CGH screening first [Miller et al., 2010]. In cases in which no abnormalities were observed, the ge- nomes of the patients were investigated by patient-parent trio whole-exome sequencing [Sandestig et al., 2020]. A second ramification could be that the phenotypic spectrum depends upon the type of NUP188 mutation. Heterozygous alterations in the NUP188 gene dosage, due to gene deletion or duplication, or loss of the C-terminal phosphorylation and ubiquitination sites required for binding to its partner proteins, provoke a dominant disorder that affects primarily the heart [Fakhro et al., 2011; Haskell et al., 2017]. In contrast, biallelic mutations close to the N-terminus of the gene, most likely leading to nonsense-mediated decay, provoke a complex NUP188 insufficiency syndrome [Sandestig et al., 2020]. The recent surge of NUP-related clinical disorders may prompt a search for NUP-mutations in the worldwide available cohorts of whole-exome sequencing data. With more case reports in hand, we may be able to devise detailed and testable hypotheses regarding the functions of NUPs.

Martin Poot

\section{References}

- Alazami AM, Patel N, Shamseldin HE Anazi S, Al-Dosari MS, et al: Accelerating novel candidate gene discovery in neurogenetic disorders via whole-exome sequencing of prescreened multiplex consanguineous families. Cell Rep 10:148-161 (2015).

Birchler JA, Veitia RA: Gene balance hypothesis: connecting issues of dosage sensitivity across biological disciplines. Proc Natl Acad Sci USA 109:14746-14753 (2012).

Bonnin E, Cabochette P, Filosa A, Jühlen R, Komatsuzaki $S$ et al: Biallelic mutations in nucleoporin NUP88 cause lethal fetal akinesia deformation sequence. PLoS Genet 14:e1007845 (2018).

Buchwalter AL, Liang Y, Hetzer MW: Nup50 is required for cell differentiation and exhibits transcription-dependent dynamics. Mol Biol Cell 25:2472-2484 (2014).

Busayavalasa K, Chen X, Östlund Farrants AK, Wagner N, Sabri N: The Nup155-mediated organisation of inner nuclear membrane proteins is independent of Nup155 anchoring to the metazoan nuclear pore complex. J Cell Sci 125:4214-4218 (2012).

Del Viso F, Huang F, Myers J, Chalfant M, Zhang $Y$, et al: Congenital heart disease genetics uncovers context-dependent organization and function of nucleoporins at cilia. Dev Cell 38: 478-492 (2016).

-Fakhro KA, Choi M, Ware SM, Belmont JW, Towbin JA, et al: Rare copy number variations in congenital heart disease patients identify unique genes in left-right patterning. Proc Natl Acad Sci USA 108:2915-2920 (2011).
Fujita A, Tsukaguchi H, Koshimizu E, Nakazato $\mathrm{H}$, Itoh $\mathrm{K}$, et al: Homozygous splicing mutation in NUP133 causes Galloway-Mowat syndrome. Ann Neurol 84:814-828 (2018).

Haskell GT, Jensen BC, Samsa LA, Marchuk D, Huang W, et al: Whole exome sequencing identifies truncating variants in nuclear envelope genes in patients with cardiovascular disease. Circ Cardiovasc Genet 10:e001443 (2017).

Hoelz A, Debler EW, Blobel G: The structure of the nuclear pore complex. Annu Rev 80:613643 (2011).

Itoh G, Sugino S, Ikeda M, Mizuguchi M, Kanno S, et al: Nucleoporin Nup188 is required for chromosome alignment in mitosis. Cancer Sci 104:871-879 (2013).

Miller DT, Adam MP, Aradhya S, Biesecker LG, Brothman AR, et al: Consensus statement: chromosomal microarray is a first-tier clinical diagnostic test for individuals with developmental disabilities or congenital anomalies. Am J Hum Genet 86:749-764 (2010).

-Miyake N, Tsukaguchi H, Koshimizu E, Shono A, Matsunaga S, et al: Biallelic mutations in nuclear pore complex subunit NUP107 cause early-childhood-onset steroid-resistant nephrotic syndrome. Am J Hum Genet 97:555566 (2015).

Nofrini V, Di Giacomo D, Mecucci C: Nucleoporin genes in human diseases. Eur J Hum Genet 24:1388-1395 (2016).

Poot M, Haaf T: Mechanisms of origin, phenotypic effects and diagnostic implications of complex chromosome rearrangements. Mol Syndromol 6:110-134 (2015).
Preston CC, Wyles SP, Reyes S, Storm EC, Eckloff BW, Faustino RS: NUP155 insufficiency recalibrates a pluripotent transcriptome with network remodeling of a cardiogenic signaling module. BMC Syst Biol 12:62 (2018).

Rossanti R, Shono A, Miura K, Hattori M, Yamamura T, et al: Molecular assay for an intronic variant in NUP93 that causes steroid resistant nephrotic syndrome. J Hum Genet 64:673679 (2019).

Rosti RO, Sotak BN, Bielas SL, Bhat G, Silhavy JL, et al: Homozygous mutation in NUP107 leads to microcephaly with steroid-resistant nephrotic condition similar to Galloway-Mowat syndrome. J Med Genet 54:399-403 (2017).

-Sandestig A, Engström K, Pepler A, Danielsson I, Odelberg-Johnssonet P et al.: NUP188 biallelic loss of function may underlie a new syndrome: nucleoporin 188 insufficiency syndrome? Mol Syndromol 10:313-319 (2020).

-Shamseldin HE, Makhseed N, Ibrahim N, Al-Sheddi T, Alobeid E, et al: NUP214 deficiency causes severe encephalopathy and microcephaly in humans. Hum Genet 138:221-229 (2019).

- Solmaz SR, Chauhan R, Blobel G, Melčák I: Molecular architecture of the transport channel of the nuclear pore complex. Cell 147:590602 (2011).

Theerthagiri G, Eisenhardt N, Schwarz H, Antonin W: The nucleoporin Nup188 controls passage of membrane proteins across the nuclear pore complex. J Cell Biol 189:1129-1142 (2010).

Wälde S, Kehlenbach RH: The part and the whole: functions of nucleoporins in nucleocytoplasmic transport. Trends Cell Biol 20:461-469 (2010). 